\title{
High dose chemotherapy and autologous hematopoietic cell transplantation for Wilms tumor: a study of the European Society for Blood and Marrow Transplantation
}

\author{
F. Spreafico ${ }^{1} \cdot$ A. Dalissier ${ }^{2} \cdot$ U. Pötschger ${ }^{3} \cdot$ F. Locatelli ${ }^{4} \cdot$ J. M. Michon ${ }^{5} \cdot$ C. Peters $\mathbb{1}^{3} \cdot$ P. Bader ${ }^{6} \cdot$ G. Bisogno $\mathbb{1}^{7} \cdot$

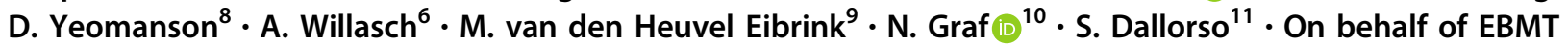 \\ Paediatric Diseases Working Party
}

Received: 5 February 2019 / Revised: 2 July 2019 / Accepted: 28 July 2019 / Published online: 18 September 2019

(c) The Author(s), under exclusive licence to Springer Nature Limited 2019

\begin{abstract}
Survival for subgroups of patients with Wilms tumor (WT), such as those who suffer from relapse, is disappointing. Some patients' treatment plans include high-dose chemotherapy (HDT) with autologous hematopoietic cell transplantation (aHCT), although proof for its benefit is lacking. To increase the level of evidence regarding children with WT receiving aHCT as consolidation of first or second remission (after first relapse), we extracted relevant data from the European Blood and Marrow Transplantation Registry concerning 69 patients. Different HDT regimens were administered, mostly either melphalan-containing $(n=34)$ or thiotepa-containing $(n=14)$. For the whole population, 5 -year overall survival (OS) and event-free survival (EFS) probabilities were $0.67( \pm 0.06)$ and $0.63( \pm 0.06)$, respectively (median observation time 7.8 years); for children transplanted in first remission, OS and EFS were $0.69( \pm 0.09)$ and $0.72( \pm 0.08)$. In univariate analysis, male gender and relapse in multiple sites were associated with lower OS probabilities. The use of a given pretransplant regimen (i.e. melphalan alone versus regimens with multiple drugs) did not seem to influence EFS/OS probability after aHCT, but significantly influenced platelet engraftment (more delayed with thiotepa). We here provide further data to improve the basis for future evidence-based clinical decision-making when using HDT and aHCT in relapsed/refractory WT.
\end{abstract}

\section{Introduction}

Wilms tumor (WT) is the most common renal cancer in childhood, with about 1000 new patients diagnosed in Europe each year [1]. With optimized use of chemotherapy, nephrectomy, and sometimes radiotherapy, a 5-year overall survival (OS) of $90 \%$ can be achieved [2-5]. About $15 \%$ of treated patients suffer from relapse of WT, mostly within 2 years after nephrectomy [6]. OS after relapse is around $50 \%$, but the outcome varies depending on several $\triangle$ F. Spreafico

filippo.spreafico@istitutotumori.mi.it

1 Deptartment of Medical Oncology and Hematology, Pediatric Unit, Fondazione IRCCS Istituto Nazionale dei Tumori, Milan, Italy

2 EBMT Paris Study Office/CEREST-TC, Department of Haematology, Saint Antoine Hospital, INSERM UMR 938, Paris, France

3 St. Anna Kinderspital, Stem Cell Transplantation Unit, Vienna, Austria

4 Department of Pediatric Hematology and Oncology, IRCCS Ospedale Pediatrico Bambino Gesù, Sapienza, University of Rome, Rome, Italy
5 Children, Adolescent and Young Adults Department, Institut Curie, Paris, France

6 Division for Stem Cell Transplantation and Immunology, Department for Children and Adolescents, University Hospital, Goethe University, Frankfurt/Main, Germany

7 Hematology Oncology Division, Department of Woman's and Child's Health, University of Padova, Padova, Italy

8 Sheffield Children's Hospital Western Bank, Sheffield, UK

9 Princess Máxima Centre for Pediatric Oncology, Utrecht, the Netherlands

10 Department of Haematology and Oncology, Saarland University Hospital, Homburg, Germany

11 IRCCS Istituto Giannina Gaslini, Genova, Italy 
prognostic factors, including initial histological risk group and tumor stage, and previous treatment intensity [7-9]. Despite fair improvement in contemporary studies, the prognosis for certain subgroups of relapsing patients, including those with high-risk histologic or molecular features, patients who had already received a number of effective drugs, or patients suffering from multiple relapses, remains dismal [9].

We have recently reported that phase I/II studies on novel agents or innovative strategies focusing on children with WT are insufficient [10], and the foundation of salvage therapy for recurrent/resistant WT mostly comes from limited and retrospective analysis [9]. We have also learned that the application of more risk-adapted retreatment strategies has improved survival to nearly $70-80 \%$ for patients who relapse after minimal first-line therapy, consisting of only vincristine and actinomycin-D, while minimizing long-term toxicity [7]. However, nearly twothirds of relapses fall into the higher risk groups, i.e., patients who have received prior treatment including doxorubicin and, sometimes, radiotherapy and additional chemotherapeutic agents; for these patients postrelapse survival rate is less than $40 \%[8,9]$. Some investigators have incorporated myeloablative high dose chemotherapy (HDT) into relapse regimens for these high-risk patients, with reported event-free survival (EFS) estimates ranging from $36 \%$ to $60 \%$ [11-15]. The value of HDT with autologous hematopoietic cell transplantation (aHCT) to treat recurrent WT is one of the unsettled questions in the field, since others report similar outcomes with standard doses of chemotherapy, $[8,16]$ but outside randomized comparison.

The comprehensive Bayesian analysis from $\mathrm{Ha}$ et al. from nonrandomized comparisons of 'no HDT' versus HDT, and from single arm studies of either 'no HDT' or HDT alone, can be regarded as the largest available attempt to summarize evidence in this field, and concluded with a deal of uncertainty concerning the role of HDT in relapsing patients [17]. Since the first European Blood and Marrow Transplant (EBMT) report, [18] the number of transplanted patients with WT included in the EBMT registry has grown to over 400 cases, most of them treated outside clinically controlled protocols. In a recent analysis from the Center for International Blood and Marrow Transplantation Research, outcomes on 253 children with relapsed WT have been described [19].

There is also a standing interest in evaluating the efficacy of HDT/aHCT in tumors refractory to standard initial treatment: this indication is even less explored, and its value has not yet been established. Metastatic diffuse anaplastic or postchemotherapy blastemal-type WTs have EFS rates largely below 30\% with conventional dose chemotherapy; therefore, the Renal Tumor Study Group of the International
Society of Pediatric Oncology is exploring the role of upfront HDT for this subgroup [20].

Recognizing this as an unmet clinical need, with the goal of improving the level of evidence on the benefit deriving from the use of HDT/aHCT in WT, especially in those patients with first complete remission (CR1) and second complete remission following first relapse (CR2), we report the results of an analysis of outcome for patients registered in the EBMT registry.

\section{Subjects and methods}

\section{Data source}

EBMT is a voluntary working group, including over 500 transplantation units that are requested to report data for all consecutive HCTs and related follow-up visits once each year. Audits are routinely performed to ensure the completeness/accuracy of data. According to EBMT rules, patients/families are requested to give informed consent for data entry into the EBMT registry database and their use for analysis in accordance with the Declaration of Helsinki.

\section{Data collection}

The present analysis concerns patients younger than 18 years, with WT in CR2 (patients relapsing after primary treatment and receiving HDT after having achieved a second complete response after first relapse) or having achieved CR1 (both patients who achieved CR after initial systemic treatment and those who failed to obtain clinical remission on initial treatment but achieved CR on secondline treatment), who have undergone a single aHCT between 1995 and 2010 at an EBMT center. Data regarding demographics, diagnosis, conditioning regimen, graft characteristics, and outcome have been obtained via a questionnaire integrating data already available in the EBMT ProMISe database. Centres were approached if they had any patients who met the above mentioned study inclusion criteria and the related patient data were properly updated in ProMISe database.

Of 414 patients with WT recorded in the EBMT Registry in the study period (1995-2010), 144 were eligible because they had received HDT and aHCT for either CR1 or CR2 disease status. Sixty-two centers (registering the 144 patients) were contacted, and 26 centers (corresponding to 79 patients) accepted to participate. A few centers answered that they did not want to participate, and some centers did not answer to our call. To note, participation was voluntary, and reasons why the centers denied or refused to take part to this study were not collected, thus this information is missing. 


\section{Statistical analysis}

The primary study endpoints were EFS and OS. Events were the first evidence post aHCT of tumor recurrence, progression, second tumor or all-cause death for EFS, and all-cause death for OS. The Kaplan-Meier method was used to estimate EFS and OS [21]. Patients with no event were censored at the date of their last follow-up evaluation. For the statistical comparison, the log-rank test and Cox-regression was used [22]. Chi-square test and Wilcoxon two-sample test was used to compare patient and transplant characteristics in CR1 and CR2 patients [23]. Median follow-up was estimated using the inverse Kaplan-Meier method.

We examined the influence of these variables on EFS and OS: patient age at transplantation ( $\leq$ or $>6$ years), anaplasia (absent versus present), gender, year of diagnosis (before or later than 2000), year of transplantation (before or later than 2000), disease status at transplantation (CR1 vs CR2), site of relapse (local, lungs only, combined local + other, other), pretransplant regimen (four options: melphalan alone, melphalan/etoposide/ carboplatin, etoposide/cyclophosphamide/thiotepa, other), stem cell source (bone marrow vs peripheral blood).

The cumulative incidence of neutrophil and platelet engraftment was estimated taking into account the competing risk of death before engraftment [24]. Estimates of the cumulative incidence of these competing events were evaluated. The statistical comparison of cumulative incidences was done using the Gray's test [25].

Statistical analyses were run with SAS ${ }^{\circledR} 9.3$ (SAS Institute, Cary, NC). Statistical significance was set at the conventional 5\% two-sided threshold.

\section{Results}

Sixty-nine patients (out of the 79) were included in this analysis because of the completeness of concerning relevant data (median age at diagnosis: 6.2 years [range: 1.9-16.1 years]). Twenty-nine patients had HDT/aHCT as CR1 consolidation, and 40 patients were transplanted in CR2 after first relapse. Table 1 shows the baseline patients' characteristics.

The preparative regimen included melphalan in 36 cases (melphalan alone in 14 cases), thiotepa in 14 cases (together with cyclophosphamide/etoposide), and other drugs in the remaining 17 cases (in two cases this information was missing). Out of 60 patients with data on radiotherapy, 28/60 cases received radiotherapy as part of the treatment strategy alongside aHCT.
Table 1 Baseline characteristics of patients

\begin{tabular}{|c|c|c|c|c|c|c|c|}
\hline & All s & ubjects & $\mathrm{CR} 1$ & & CR2 & deaths & $p$-value \\
\hline \multirow[t]{2}{*}{ Age at aHCT, median (range) } & \multicolumn{2}{|c|}{$\begin{array}{l}6.2 \text { years } \\
(1.9-16.1)\end{array}$} & \multicolumn{2}{|c|}{$\begin{array}{l}5.9 \\
(1.9-11.2)\end{array}$} & \multicolumn{2}{|c|}{$\begin{array}{l}5.3 \\
(3.5-16.1)\end{array}$} & \multirow[t]{2}{*}{0.286} \\
\hline & $n$ & $\%$ & $n$ & $\%$ & $n$ & $\%$ & \\
\hline Overall series & & 69 & & 29 & & 40 & \\
\hline \multicolumn{8}{|l|}{ Gender } \\
\hline Male & 34 & $49 \%$ & 15 & $52 \%$ & 19 & $48 \%$ & \multirow[t]{2}{*}{0.729} \\
\hline Female & 35 & $51 \%$ & 14 & $48 \%$ & 21 & $53 \%$ & \\
\hline \multicolumn{8}{|l|}{ Draft type } \\
\hline Bone marrow & 9 & $13 \%$ & 5 & $17 \%$ & 4 & $10 \%$ & \multirow[t]{2}{*}{0.378} \\
\hline PBSC & 60 & $87 \%$ & 24 & $83 \%$ & 36 & $90 \%$ & \\
\hline \multicolumn{8}{|l|}{ Conditioning regimen groups } \\
\hline Melphalan only & 14 & $21 \%$ & 6 & $21 \%$ & 8 & $21 \%$ & \multirow[t]{5}{*}{0.273} \\
\hline MEC & 22 & $33 \%$ & 9 & $31 \%$ & 13 & $34 \%$ & \\
\hline Thiotepa-containing & 14 & $21 \%$ & 9 & $31 \%$ & 5 & $13 \%$ & \\
\hline Other & 17 & $25 \%$ & 5 & $17 \%$ & 12 & $32 \%$ & \\
\hline Missing & 2 & - & 0 & - & 2 & - & \\
\hline \multicolumn{8}{|l|}{ Radiation therapy at aHCT } \\
\hline No & 32 & $53 \%$ & 12 & $50 \%$ & 20 & $56 \%$ & \\
\hline Yes & 28 & $47 \%$ & 12 & $50 \%$ & 16 & $44 \%$ & \\
\hline Missing & 9 & - & 5 & - & 4 & - & \\
\hline \multicolumn{8}{|l|}{ Histological subtype } \\
\hline Non anaplastic & 54 & $92 \%$ & 21 & $88 \%$ & 33 & $94 \%$ & \multirow[t]{3}{*}{0.358} \\
\hline Anaplastic & 5 & $8 \%$ & 3 & $13 \%$ & 2 & $6 \%$ & \\
\hline missing & 10 & - & 5 & - & 5 & - & \\
\hline \multicolumn{8}{|l|}{ Year of diagnosis } \\
\hline$<2000$ & 30 & $43 \%$ & 15 & $52 \%$ & 15 & $38 \%$ & \multirow[t]{2}{*}{0.239} \\
\hline$\geq 2000$ & 39 & $57 \%$ & 14 & $48 \%$ & 25 & $63 \%$ & \\
\hline \multicolumn{8}{|l|}{ Year of aHCT } \\
\hline$<2000$ & 20 & $29 \%$ & 10 & $34 \%$ & 10 & $25 \%$ & \multirow[t]{2}{*}{0.392} \\
\hline$\geq 2000$ & 49 & $71 \%$ & 19 & $66 \%$ & 30 & $75 \%$ & \\
\hline \multicolumn{8}{|l|}{ Site of relapse } \\
\hline Lungs only & 22 & $63 \%$ & - & - & 22 & $63 \%$ & \multirow[t]{5}{*}{-} \\
\hline Lungs + other & 3 & $9 \%$ & - & - & 3 & $9 \%$ & \\
\hline Other & 5 & $14 \%$ & - & - & 5 & $14 \%$ & \\
\hline Local & 5 & $14 \%$ & - & - & 5 & $14 \%$ & \\
\hline $\mathrm{NA} /$ missing & 34 & - & 29 & - & 5 & - & \\
\hline \multicolumn{8}{|l|}{ Metastases at diagnosis } \\
\hline Lungs only & 28 & $49 \%$ & 11 & $52 \%$ & 17 & $47 \%$ & \multirow[t]{6}{*}{0.796} \\
\hline Lung + other & 6 & $11 \%$ & 2 & $10 \%$ & 4 & $11 \%$ & \\
\hline Nodes & 3 & $5 \%$ & 2 & $10 \%$ & 1 & $3 \%$ & \\
\hline Other & 3 & $5 \%$ & 1 & $5 \%$ & 2 & $6 \%$ & \\
\hline None & 17 & $30 \%$ & 5 & $24 \%$ & 12 & $33 \%$ & \\
\hline Missing & 12 & - & 8 & - & 4 & - & \\
\hline Median observation time & \multicolumn{2}{|c|}{7.8 years } & 6.5 & years & 10.2 & years & \\
\hline
\end{tabular}

$C R 1$ first complete remission, $C R 2$ second complete remission, $a H C T$, autologous hematopoietic cell transplantation, $M E C$ melphalan/etoposide/carboplatin regime, $N A$ not available

\section{Survival analysis}

The median follow-up was 7.8 years (range, 6.5-10.2 years). All the 23 deaths recorded were tumor related (no toxic deaths occurred). For the whole series, the 5-year EFS and OS probabilities were $0.63( \pm 0.06)$ and $0.67( \pm 0.06)$, respectively (Fig. 1). 


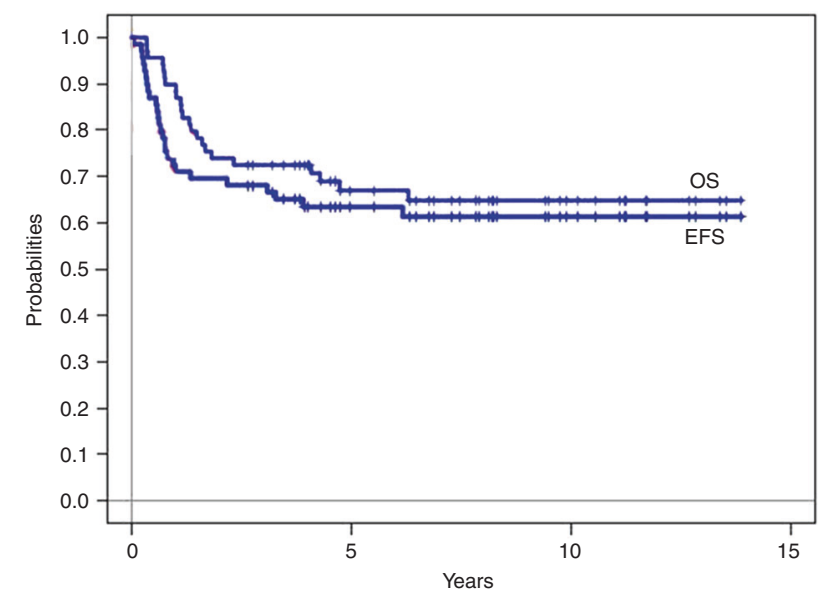

Fig. 1 For the whole series, the 5-year EFS and OS probabilities were $0.63( \pm 0.06)$ and $0.67( \pm 0.06)$, respectively

Univariate analyses on EFS and OS probabilities showed worse outcomes for male patients (for both endpoints), relapse in combined multiple sites as compared with lung alone (only for OS), the use of peripheral blood as stem cell source (both endpoints), but not for the other studied variables.

Five children out of $69(8 \%)$ had diffuse anaplastic tumors, and one death occurred among them (after second tumor relapse).

Survival analysis on EFS and OS showed lower probabilities for CR2 patients (EFS: $0.59[ \pm 0.08]$; OS: 0.62 $[ \pm 0.08]$ ), as compared with CR1 (for EFS: $0.69[ \pm 0.09]$, $p=0.328$; for OS: $0.72[ \pm 0.08], p=0.573$ ), which was not statistically significant. We evaluated the potential influence of the drugs used in the pretransplant regimens, and statistically different survival probabilities (for both endpoints) were not observed. EFS analysis showed noninferior probability with using melphalan alone $(0.79 \pm 0.11)$.

Table 2 shows the outcomes, for each endpoint, in terms of 5-year survival probabilities considering the studied factors.

\section{Hematopoietic recovery}

The day-30 cumulative incidence of platelet engraftment was significantly worse for patients receiving aHCT before year $2000(35 \pm 11 \%)$ compared with patients treated later $(67 \pm 7 \%)(p=0.03)$, and when thiotepacontaining regime was used $(14 \pm 9 \%)$ as compared with melphalan alone $(64 \pm 13 \%)$ or melphalan-containing regimens $(77 \pm 9 \%)(p=0.001)$. Other studied variables (including stem cell source) were not associated with a significantly different platelet day-30 engraftment. No variables were associated with a significantly different neutrophil day-30 or day-60 engraftment.

\section{Discussion}

There is increasing awareness that patients with relapsed WT have heterogeneous outcomes. A number of potential prognostic features have been evaluated; anaplastic or SIOP blastemal-type histology and initial chemotherapy including doxorubicin are the two features that have been consistently associated with worse outcome $[9,17]$. There is a consensus that initial treatment received (which in turn reflects primary tumor histology, stage and biological variables) is a key factor in subsequent stratification for relapse therapy, which should include drugs not used in frontline treatment. In the more up-to-date treatment guideline of the SIOP Umbrella protocol, a standardized relapse treatment is proposed according to three risk categories [20]. The standard-risk group includes patients with favorable histology WT who relapse after vincristine and actinomycin-D chemotherapy [7, 15, 17]. They will receive a four-drug regimen (doxorubicin, carboplatin, etoposide, cyclophosphamide), and survival rates are expected to be $70-80 \%$, [20] The high-risk group includes patients with favorable histology WT who relapse after therapy with three or more agents. Survival rates for these patients are expected to be $40-50 \%[8,9,15]$. The veryhigh-risk group includes patients with recurrent anaplastic or blastemal-type WT. These patients have a dismal survival in the $10 \%$ range, with very poor responses to any drug or combination, which is likely due to intrinsic drug resistance. These last two groups of patients represent a subset of children with a significant risk of death from WT, thus their clinical needs include consideration of novel drug development and treatment strategy optimization, such as determination of the optimum intensity of retreatment [26-29]. Given their unsatisfactory outcome, some investigators have used HDT and aHCT with the aim of improving survival rates [11-15].

The proportion of patients transplanted for recurrent WT seems to have increased over time, $[18,19]$ although randomized comparison of the potential additional benefit of such an approach over systematically intensifying nonmyeloablative chemotherapy has never been investigated. We know that evidence for efficacy of HDT with aHCT in patients experiencing relapse after treatment for their WT is limited. Prospective evaluation of the role of HDT/ aHCT has been extensively discussed, but not attempted because such a randomized phase III trial should need to be run for an unreasonable period of time even with international cooperation, and appropriate funding [30]. Thus the question of summarizing the current information on the merits of an HDT approach for these patients remains relevant.

In this study, we have described the OS and EFS of patients with WT reported to the EBMT registry, with 
Table 2 The table shows the outcomes, for each endpoint, in terms of 5-year survival probabilities considering the studied factors

\begin{tabular}{|c|c|c|c|c|c|c|c|}
\hline & \multirow[b]{2}{*}{ N. of patients } & \multicolumn{3}{|l|}{ OS } & \multicolumn{3}{|l|}{ EFS } \\
\hline & & N. of events & probability & $p$-value & N. of events & probability & $p$-value \\
\hline All patients & 69 & 23 & $0.67 \pm 0.06$ & & 26 & $0.63 \pm 0.06$ & \\
\hline \multicolumn{8}{|l|}{ Gender } \\
\hline Male & 34 & 15 & $0.54 \pm 0.09$ & \multirow[t]{2}{*}{0.048} & 17 & $0.49 \pm 0.06$ & \multirow[t]{2}{*}{0.041} \\
\hline Female & 35 & 8 & $0.79 \pm 0.07$ & & 9 & $0.77 \pm 0.07$ & \\
\hline \multicolumn{8}{|l|}{ Age at aHCT } \\
\hline$\leq 6$ years & 30 & 7 & $0.77 \pm 0.08$ & \multirow[t]{2}{*}{0.202} & 8 & $0.77 \pm 0.08$ & \multirow[t]{2}{*}{0.127} \\
\hline$>6$ years & 39 & 16 & $0.59 \pm 0.08$ & & 18 & $0.53 \pm 0.08$ & \\
\hline \multicolumn{8}{|l|}{ Anaplasia } \\
\hline Absent & 54 & 15 & $0.72 \pm 0.06$ & \multirow[t]{3}{*}{0.711} & 18 & $0.68 \pm 0.06$ & \multirow[t]{3}{*}{0.610} \\
\hline Present & 5 & 1 & $0.80 \pm 0.18$ & & 1 & $0.80 \pm 0.18$ & \\
\hline $\mathrm{Na}$ & 2 & 0 & $1.00 \pm 0.00$ & & 0 & $1.00 \pm 0.00$ & \\
\hline \multicolumn{8}{|c|}{ Year of diagnosis } \\
\hline$<2000$ & 30 & 14 & $0.53 \pm 0.09$ & \multirow[t]{2}{*}{0.07} & 14 & $0.53 \pm 0.09$ & \multirow[t]{2}{*}{0.148} \\
\hline$\geq 2000$ & 39 & 9 & $0.79 \pm 0.07$ & & 12 & $0.71 \pm 0.07$ & \\
\hline \multicolumn{8}{|l|}{ Year of aHCT } \\
\hline$<2000$ & 20 & 10 & $0.49 \pm 0.11$ & \multirow[t]{2}{*}{0.08} & 10 & $0.50 \pm 0.11$ & \multirow[t]{2}{*}{0.101} \\
\hline$\geq 2000$ & 49 & 13 & $0.74 \pm 0.06$ & & 16 & $0.69 \pm 0.07$ & \\
\hline \multicolumn{8}{|c|}{ Disease status at aHCT } \\
\hline CR1 & 29 & 9 & $0.72 \pm 0.08$ & \multirow[t]{2}{*}{0.573} & 9 & $0.69 \pm 0.09$ & \multirow[t]{2}{*}{0.328} \\
\hline CR2 & 40 & 14 & $0.62 \pm 0.08$ & & 17 & $0.59 \pm 0.08$ & \\
\hline \multicolumn{8}{|c|}{ Metastasis at diagnosis } \\
\hline Lungs only & 28 & 6 & $0.79 \pm 0.08$ & \multirow[t]{5}{*}{0.948} & 7 & $0.75 \pm 0.08$ & \multirow[t]{5}{*}{0.912} \\
\hline Lung + other & 6 & 2 & $0.63 \pm 0.21$ & & 2 & $0.67 \pm 0.19$ & \\
\hline Nodes only & 3 & 1 & $0.67 \pm 0.27$ & & 1 & $0.67 \pm 0.27$ & \\
\hline Other & 3 & 1 & $0.67 \pm 0.27$ & & 1 & $0.67 \pm 0.27$ & \\
\hline None & 17 & 5 & $0.69 \pm 0.12$ & & 7 & $0.65 \pm 0.12$ & \\
\hline \multicolumn{8}{|l|}{ Relapse site } \\
\hline Lungs only & 22 & 5 & $0.72 \pm 0.11$ & 0.030 & 8 & $0.67 \pm 0.10$ & 0.081 \\
\hline Lungs & 3 & 2 & $0.33 \pm 0.27$ & & 2 & $0.33 \pm 0.27$ & \\
\hline Other & 5 & 0 & $1.00 \pm 0.00$ & & 0 & $1.00 \pm 0.00$ & \\
\hline Local & 5 & 3 & $0.40 \pm 0.22$ & & 3 & $0.40 \pm 0.22$ & \\
\hline Conditioning dru & & & & & & & \\
\hline Melphalan & 14 & 3 & $0.79 \pm 0.11$ & 0.493 & 3 & $0.79 \pm 0.11$ & 0.265 \\
\hline MEC & 22 & 10 & $0.57 \pm 0.11$ & & 12 & $0.50 \pm 0.11$ & \\
\hline CyET & 14 & 4 & $0.71 \pm 0.12$ & & 5 & $0.64 \pm 0.13$ & \\
\hline Other & 17 & 5 & $0.68 \pm 0.12$ & & 5 & $0.70 \pm 0.11$ & \\
\hline Stem cell source & & & & & & & \\
\hline $\mathrm{BM}$ & 9 & 0 & $1.00 \pm 0.00$ & 0.038 & 0 & $1.00 \pm 0.00$ & 0.024 \\
\hline PB & 60 & 23 & $0.62 \pm 0.06$ & & 26 & $0.58 \pm 0.06$ & \\
\hline
\end{tabular}

$O S$ overall survival, EFS event-free survival, $C R 1$ first complete remission, $C R 2$ second complete remission, $M E C$ melphalan/etoposide/ carboplatin, $C y E T$ cyclophosphamide/etoposide/thiothepa, $B M$ bone marrow; $P B$ peripheral blood, $a H C T$ autologous hematopoietic stem cell transplantation

the purpose of examining the potential benefit of HDT and aHCT in specific disease settings, including the frontline use of HDT in patients with features of high-risk disease.
The best evidence for selecting one putative superior approach comes from the work by $\mathrm{Ha}$ et al. [17], where information from non-randomized comparisons of "no HDT' versus HDT, and from single arm studies of either 
'no HDT' or HDT alone, were synthesized through a Bayesian framework. From this analysis, estimates of 3 -year EFS varied considerably with a median of $52 \%$ for those receiving HDT and $52.5 \%$ for 'no HDT' (the corresponding values for OS were $60.9 \%$ and $52.9 \%$, respectively). Ha et al. concluded that the evidence suggested some value of HDT and aHCT for patients with the highest risk features at relapse, but recognized a great deal of uncertainty concerning the remaining patients, and identified the need to gain additional evidence.

In a recent paper, Malogolowkin et al. [19] retrospectively examined a large series of patients $(n=253)$ treated with HDT for WT reported to the Center for International Blood and Marrow Transplantation Research Registry. The EFS and OS estimates were $36 \%$ and $45 \%$, respectively, which are likely below the expected benefit from HDT. Patients included in the analysis were very heterogeneous in terms of disease status at transplant, which might render it difficult to interpret their results, and emphasizes the need to selectively focus on subgroup analysis. Of note, $25 \%$ of patients were transplanted without having achieved $\mathrm{CR}$, which is regarded as an adverse factor for outcome after aHCT, and a significant proportion of patients were transplanted in $\geq \mathrm{CR} 2$ remission status.

The results of our study are not different to those observed in previous reports with similar endpoints (reviewed in $\mathrm{Ha}$ et al.). One main limitation lies in its retrospective nature, being a registry-based analysis, and consequently many information were not available or partially reported. Specifically related to patients receiving HDT as consolidation of first remission (CR1), an unexplored yet significant issue, we recognize that it would have been important to better characterize the group. These patients may have received HDT because of slowly responding disease (thus regarded as refractory to conventional initial chemotherapy), or because they were judged to be at high risk of relapse by the treating physician (unfortunately the 'reason' for CR1 consolidation was not expressly reported). Despite these difficulties, we have attempted a synthesis of the relevant information but remain conscious of the limitations this imposes on our conclusions. Identification of more efficacious drugs or strategies for those tumors with initial high-risk histology (anaplastic or pre-treated blastemal-type WTs) or adverse molecular features (like 1q gain or loss of heterozygosity at chromosomes $1 p$ and/or $16 q$ ), known to be refractory to first-line conventional therapies is regarded as an important need for WT $[20,26]$. Noteworthy, 4 out of 5 patients with anaplasia transplanted (three cases while in CR1, and two cases in CR2) are alive, implying some benefit deriving from aHCT in this setting. Keeping in mind the problem of obtaining some response before aHCT is administered as consolidation in patients with refractory or relapsed anaplastic tumor, these few data seem to support the possibility that HDT may overcome the intrinsic resistance to drugs inherent to P53 alterations observed in anaplastic tumors [31].

Survival results for patients with CR1 or CR2 status were not statistically different, yet a comparison between these two groups of patients is challenging because of very different clinical characteristics, and beyond the remit of this study. Nevertheless, it is noteworthy to underscore that patients transplanted after remission of relapsed disease (CR2) represent a group of children with recurrent disease associated with more favorable features, including chemosensitiveness, and worthy of consideration for intensified chemotherapy approaches.

Given that most relapsed WT patients are already at risk of renal impairment and will be receiving nephrotoxic drugs for initial treatment of relapse, then there is an important question about which regimen is better tolerated, while maintaining efficacy. To date there is not a consensus on the optimal conditioning regimen in this setting, and a wide range of different drugs and combination has been used $[17,19]$. Our data seems to support the use of melphalan alone for HDT, which led to noninferior survival rates compared with other drugs or drug combinations (noteworthy, 79\% both for EFS and OS), and to better engraftment comparing to thiotepa-containing regimens. This information is going to improve the decision-making process when selecting the most suitable pretransplant regimen for aHCT.

Univariate analysis appearing to show worse EFS and OS rates when peripheral blood was used as stem cell source are difficult to explain, especially as all deaths were tumor related, and we would not recommend that these retrospective data be used to influence decisions regarding selection of technique for stem cell harvest.

Updated evidence from the studies like ours, together with any emerging studies, will form the basis for future evidence-based clinical decision-making. Most importantly, since no comprehensive clinical studies on recurrent disease are currently active, our results may support clinicians when deciding how to treat a child suffering from WT relapse.

Acknowledgements List of contributing centers: IRRCS Ospedale Pediatrico Bambino Gesù Rome; Institute Curie Paris; University of Helsinki Hospital for Children \& Adolescents; Clinica di Oncoematologia Pediatrica Dipartimento di Pediatria Padova; Our Lady's Children's Hospital Crumlin Dublin; Hebron hospital Barcelona; North Trent BMT Programme Sheffield Teaching Hospitals NHS Trust/Sheffield Children's Hospital; Cape of Hope Wroclaw Medical University; Klinikum der Johann-Wolfgang Goethe Universitaet Frankfurt_am_Main; University Hospital Bern Paediatric Hematology/ Oncology Bern; Inst. Português de Oncologia do Porto BMT Unit; Bristol Royal Hospital for Children Dept. of Paediatric Oncology/ BMT; St. Anna Kinderspital Stem Cell Transplantation Unit Vienna; 
Edmond \& Lily Safra Children's Hospital Paediatric Hemato Oncology \& BMT Tel-Hashomer; Pediatric University Teaching Hospital BMT Unit II Children's Clinic Bratislava; Service d'Hématologie et d'Oncologie pédiatrique CHU Hautepierre Strasbourg; Turku University Hospital TD7 (Stem Cell Transplant Unit); Chu Estaing Service d'hématologie clinique Adulte et pédiatrie Clermont, University Hospital "Queen Johanna-Isul" Sofia, Poznan University of Medical Sciences Department of Pediatric Onology, Hematology \& HSCT, The Children's Hospital at Westmead Sydney; Clínica Universitaria de Navarra Pamplona; Pediatric Oncology Unit, Fondazione IRCCS Istituto Nazionale dei Tumori, Milano, Italy; Istituto Giannina Gaslini, Genova, Italy.

\section{Compliance with ethical standards}

Conflict of interest The authors declare that they have no conflict of interest.

Publisher's note Springer Nature remains neutral with regard to jurisdictional claims in published maps and institutional affiliations.

\section{References}

1. Gatta G, Botta L, Rossi S, Aareleid T, Bielska-Lasota M, Clavel J, et al. Childhood cancer survival in Europe 1999-2007: results of EUROCARE-5 a population-based study. Lancet Oncol. 2014; $15: 35-47$.

2. Graf N, Van Tinteren H, Bergeron C, Pein F, van den HeuvelEibrink MM, Sandstedt B, et al. Characteristics and outcome of stage II and III non-anaplastic Wilms' tumour treated according to the SIOP trial and study 93-01. Eur J Cancer. 2012;48:3240-48.

3. Pritchard-Jones K, Bergeron C, de Camargo B, van den HeuvelEibrink MM, Acha T, Godzinski J, et al. Omission of doxorubicin from the treatment of stage II-III, intermediate-risk Wilms' tumour (SIOP WT 2001): an open-label, non-inferiority, randomised controlled trial. Lancet. 2015;386:1156-64.

4. Gratias EJ, Dome JS, Jennings LJ, Chi YY, Tian J, Anderson J, et al. Association of chromosome 1q gain with inferior survival in favorable-histology Wilms Tumor: a report from the Children's Oncology Group. J Clin Oncol. 2016;34:3189-94.

5. Spreafico F, Biasoni D, Lo Vullo S, Gandola L, D'Angelo P, Terenziani M, et al. Results of the third AIEOP cooperative protocol on Wilms tumor (TW2003) and related considerations. J Urol. 2017;5:1138-45.

6. Brok J, Lopez-Yurda M, Tinteren HV, Treger TD, Furtwängler R, Graf N, et al. Relapse of Wilms' tumour and detection methods: a retrospective analysis of the 2001 Renal Tumour Study GroupInternational Society of Paediatric Oncology Wilms' tumour protocol database. Lancet Oncol. 2018;19:1072-81.

7. Green DM, Cotton CA, Malogolowkin M, Breslow NE, Perlman E, Miser J, et al. Treatment of Wilms tumor relapsing after initial treatment with vincristine and actinomycin D. A report from the National Wilms Tumor Study Group. Pediatr Blood Cancer. 2007;48:493-9.

8. Malogolowkin M, Cotton CA, Green DM, Breslow NE, Perlman E, Miser J, et al. Treatment of Wilms tumor relapsing after initial treatment with vincristine, actinomycin $\mathrm{D}$, and doxorubicin. A report from the National Wilms Tumor Study Group. Pediatr Blood Cancer. 2008;50:236-41.

9. Spreafico F, Pritchard-Jones K, Malogolowkin MH, Bergeron C, Hale J, de Kraker J, et al. Treatment of relapsed Wilms tumors: lessons learned. Expert Rev Anticancer Ther. 2009;9:1807-15.
10. Brok J, Pritchard-Jones K, Geller JI, Spreafico F. Review of phase I and II trials for Wilms' tumour-can we optimise the search for novel agents? Eur J Cancer. 2017;79:205-13.

11. Pein F, Michon J, Valteau-Couanet D, Quintana E, Frappaz D, Vannier JP, et al. High-dose melphalan, etoposide, and carboplatin followed by autologous stem-cell rescue in pediatric high-risk recurrent Wilms' tumor: a French Society of Pediatric Oncology study. J Clin Oncol. 1998;16:3295-301.

12. Kremens B, Gruhn B, Klingebiel T, Hasan C, Laws HJ, Koscielniak E, et al. High-dose chemotherapy with autologous stem cell rescue in children with nephroblastoma. Bone Marrow Transpl. 2002;30:893-8.

13. Campbell AD, Cohn SL, Reynolds M, Seshadri R, Morgan E, Geissler G, et al. Treatment of relapsed Wilms' tumor with highdose therapy and autologous hematopoietic stem-cell rescue: the experience at Children's Memorial Hospital. J Clin Oncol. 2004;22:2885-90.

14. Spreafico F, Bisogno G, Collini P, Jenkner A, Gandola L, D'Angelo P, et al. Treatment of high-risk relapsed Wilms tumor with dose-intensive chemotherapy, marrow-ablative chemotherapy, and autologous hematopoietic stem-cell support: experience by the Italian Association of Pedaitric Hematology and Oncology. Pediatr Blood Cancer. 2008;51:23-8.

15. Hale J, Hobson R, Moroz V, Sartori P. Results of UK Children's Cancer and Leukemia Group (CCLG) protocol for relapsed Wilms tumor (UKWR): unified relapse strategy improves outcome. In: 40th meeting of International Society of Paediatric Oncology. 2008;O154:62.

16. Abu-Ghosh AM, Krailo MD, Goldman SC, Slack RS, Davenport $\mathrm{V}$, Morris E, et al. Ifosfamide, carboplatin and etoposide in children with poor-risk relapsed Wilms' tumor: a Children's Cancer group report. Ann Oncol. 2002;13:460-9.

17. Ha TC, Spreafico F, Graf N, Dallorso S, Dome JS, Malogolowkin M, et al. An international strategy to determine the role of high dose therapy in recurrent Wilms' tumour. Eur J Cancer. 2013;49:194-210.

18. Garaventa A, Hartmann O, Bernard JL, Zucker JM, Pardo N, Castel V, et al. Autologous bone marrow transplantation for pediatric Wilms' tumour: the experience of the European Bone Marrow Transplantation Solid Tumour Registry. Med Pediatr Oncol. 1994;22:11-4.

19. Malogolowkin MH, Hemmer MT, Le-Rademacher J, Hale GA, Metha PA, Smith AR, et al. Outcomes following autologous hematopoietic stem cell transplant for patients with relapsed Wilms' tumor: a CIBMTR retrospective analysis. Bone Marrow Transpl. 2017;52:1549-55.

20. van den Heuvel-Eibrink MM, Hol JA, Pritchard-Jones $K$, van Tinteren H, Furtwängler R, Verschuur AC, et al. Position paper: rationale for the treatment of Wilms tumour in the UMBRELLA SIOP-RTSG 2016 protocol. Nat Rev Urol. 2017;14: 743-52.

21. Kaplan EL. Nonparametric estimation from incomplete observations. J Am Stat Assoc. 1958;53:457-81.

22. Cox D. Regression models and life table. J R Stat Soc Ser B Stat Methodol. 1972;34:187-220.

23. Wilcoxon F. Individual comparisons by ranking methods. Biom Bull. 1945;6:80-3.

24. Kalbfleisch JD, Prentice RL. Wiley Series in Probability and Statistics. The statistical analysis of failure time data. 2nd ed. 2002; John Wiley and Sons.

25. Gray RJ. A class of k-sample tests for comparing the cumulative incidence of a competing risk. Ann Stat. 1988;16:1141-54.

26. Dome JS, Graf N, Geller JI, Fernandez CV, Mullen EA, Spreafico $\mathrm{F}$, et al. Advances in Wilms tumor treatment and biology: progress through international collaboration. J Clin Oncol. 2015;33:2999-3007. 
27. Mavinkurve-Groothuis AM, van den Heuvel-Eibrink MM, Tytgat GA, van Tinteren H, Vujanic G, Pritchard-Jones KL, et al. Treatment of relapsed Wilms tumour (WT) patients: experience with topotecan. A report from the SIOP Renal Tumour Study Group (RTSG). Pedia Blood Cancer. 2015;62: 598-602.

28. Hol JA, van den Heuvel-Eibrink MM, Graf N, Pritchard-Jones K, Brok $\mathrm{J}$, van Tinteren $\mathrm{H}$, et al. Irinotecan for relapsed Wilms tumor in pediatric patients: SIOP experience and review of the literature-A report from the SIOP Renal Tumor Study Group. Pediatr Blood Cancer. 2018;65. https://doi.org/10.1002/pbc. 26849.
29. Dome JS, Liu T, Krasin M, Lott L, Shearer P, Daw NC, et al. Improved survival for patients with recurrent Wilms tumor: the experience at St. Jude Children's Research Hospital. Hosp J Pediatr Hematol/Oncol. 2002;24:192-8.

30. Spreafico F, Pritchard-Jones K, Bergeron C, de Kraker, Dallorso S, Graf N. Value and difficulties of a common European strategy for recurrent Wilms' tumor. Expert Rev Anticancer Ther. 2009;9:693-6.

31. Wegert J, Vokuhl C, Ziegler B, Ernestus K, Leuschner I, Furtwängler R, et al. TP53 alterations in Wilms tumour represent progression events with strong intratumour heterogeneity that are closely linked but not limited to anaplasia. J Pathol Clin Res. 2017;3:234-48. 